East African Medical Journal Vol. 86 No. 7 August 2009

HEPATITIS E VIRUS INFECTION AMONG PIG HANDLERS IN ACCRA, GHANA

A. A. Adjei, PhD, Department of Pathology, J. T. Aviyase, Dip., BSc, Department of Microbiology, Y. Tettey, MBChB, FWACP, Department of Pathology, University of Ghana Medical School, College of Health Sciences, Accra, Ghana, C. Adu-Gyamfi, Dip., Komfo Anokye Teaching Hospital, Kumasi, Ghana, J. A. A. Mingle, PhD, P. F. Ayeh-Kumi,PhD, T. K. Adiku, PhD, Department of Microbiology and R. K. Gyasi, MBChB, FWACP, Department of Pathology, University of Ghana Medical School, College of Health Sciences, Accra, Ghana

Request for reprints to: Prof. A. A. Adjei, Department of Pathology, University of Ghana Medical School, College of Health Sciences, Accra, Ghana

\title{
HEPATITIS E VIRUS INFECTION AMONG PIG HANDLERS IN ACCRA, GHANA
}

\author{
A. A. ADJEI, J. T. AVIYASE, Y. TETTEY, C. ADU-GYAMFI, J. A. A. MINGLE, \\ P. F. AYEH-KUMI, T. K. ADIKU, and R. K. GYASI
}

\begin{abstract}
Objective: To determine the correlates of hepatitis E virus infection (HEV) in a sample of persons who work with pigs.

Design: Cross-sectional study.

Setting: Three pig farms in the Greater Accra Region of Ghana.

Subjects: Persons who work with pigs seen at the selected pig farms between the months of January and May 2008.

Results: One hundred and five persons who work with pigs voluntarily completed a risk-factor questionnaire and provided blood samples for unlinked anonymous testing for the presence of antibodies to HEV. The median age of participants was $36.5 \pm 15.0$ years (range 12-65 years). Of the 105 subjects tested, HEV seroprevelance was $38.1 \%$. On multivariate analysis, the independent determinants of HEV infection were being employed on the farm for less than six months (odds ratio (OR) 9.1; 95\% confidence interval $(95 \% \mathrm{CI}) \mathbf{1 . 0 - 8 1 . 4}$ and having piped water in the household and/or on the farm (OR 3.9; 95\% CI 0.4-90.8).

Conclusion: Consistent with similar studies worldwide, the results of our studies revealed a high prevalence of HEV infection in persons who work with pigs. Further studies need to be done to isolate, characterise the virus and define the clinical and epidemiological significance of HEV infection in this population.
\end{abstract}

\section{INTRODUCTION}

Hepatitis E virus (HEV) infection is a major cause of human viral disease with clinical and pathological features of acute hepatitis. The infection represents an important publichealth concern in many developing countries, where it is primarily transmitted through the faecal oral route due to contaminated water and food (1), and is often responsible for epidemic outbreaks (2). The infection affects primarily young adults and is generally mild, except for women in late pregnancy in whom $20 \%$ mortality has been reported (3). The first animal strain of HEV was characterised in pigs in the United States of America $(4,5)$ and since then several other strains have been described in pigs worldwide $(5,6)$ suggestive that pigs can represent a reservoir of the infection. The identification of a USA strain of HEV apparently acquired inside the USA after the isolation of a closely related HEV strain from swine in the same region of the USA validates that $\mathrm{HEV}$ is a zoonotic infection $(4,5)$. Similar findings have been reported in China (7), South Korea (8) and Japan (9).

Growing evidence suggests that individuals who work in contact with swine such as pig farmers, veterinarians and slaughterhouse workers are at increased risk of acquiring HEV infection (10-12). To our knowledge, there is no documented information or data on the prevalence of HEV in Ghana, despite anecdotal reports of increased non-A and non-B hepatitis (Unpublished report, Department of Medicine and Therapeutics, Korle-Bu Teaching Hospital, Accra, Ghana) among patients with human viral hepatitis seen at the Department.

HEV infection is likely to be prevalent in Ghana for two reasons. First, various animals that are potential sources of transmission (pigs, sheep, goats, and cattle) share a habitat with humans. Second, the common sources of drinking water, including tap water, may be contaminated because of the inadequacy of standard water treatment measures to remove the organism. Here we report the results 
of our 5-month study of the prevalence of anti-HEV antibodies among persons who work with pigs. In this study, we have also examined the association of HEV with various suggested risk factors for its transmission.

\section{MATERIALS AND METHODS}

Study setting and subjects: This study was conducted among workers in three commercial pig farms in the Greater Accra Region of Ghana. The pig rearing facilities used for the study ranged from small family-run piggeries (200 pigs) to large-scale pig farming operations (4000 pigs) where animal housing conditions, sanitation and overall management were generally of a lower standard. All the farms are situated within the communities in high population density areas. Two of the farms are close to each other while the third farm is about $65 \mathrm{~km}$ from the other two farms.

Subjects for the study were both male and female workers of the farms. The study population was of similar socio-economic and cultural backgrounds. In general, participants had been residing in their respective communities for most part of their lives. Farming is the major source of income; most farmers rear pigs, and other domestic animals such as goats, sheep, cows and poultry for their own consumption and for sale to supplement the family incomes. After an explanation of the purpose of the study, all the workers were invited to participate. They were informed that the study was confidential and that information provided by them would not affect their employment status. A total of 105 persons joined in the research. Written and informed consent was obtained from each participant, and the information regarding the protocol and informed consent was presented at the appropriate literacy level. The study was conducted in a confidential manner and random unique study generated numbers were employed to identify the participants.

Ethical consideration: The study was reviewed and approved by the Ethical and Protocol Review Committee of the University of Ghana MedicalSchool, Accra, Ghana.

Design: This was a cross-sectional study conducted between the months of January and May 2008.

Questionnaire: All the 105 consenting participants completed a structured questionnaire assessing sociodemographic characteristics, and a risk factor profile for the infection under investigation.

Sample collection and serological tests: Blood $(10 \mathrm{ml})$ samples were collected from each of the consenting participants in plain tubes. Samples were centrifuged and the sera kept frozen at $20^{\circ} \mathrm{C}$ until analysed. Sera were tested at the Public Health Reference Laboratories, Korle-Bu, Accra, Ghana, for the presence of IgM and IgG antibodies (using ELISA Kit produced by International Immuno-Diagnostics, CA, USA) to HEV, in accordance with the manufacturer's instructions. The results were scored as positive or negative according to the standard procedures recommended by the manufacturer. Positive and negative controls were included in all the ELISA microplates assayed.

Statistical analysis: The Statistical Analysis Software (SAS Institute, Cary, NC, USA) version 9.1 was used to complete all data analyses. For each generally accepted risk factor for HEV infection, the odds ratio (OR) and the 95\% confidence interval (95\% CI) were calculated to assess associations with sociodemographic and behavioural variables in bivariate analysis. A P-value of $<0.05$ was considered significant. Independent determinants of anti-HEV reactivity were evaluated by calculating the adjusted OR by multivariate analysis for the risk factor variables found to be significant and to estimate their joint influence.

\section{RESULTS}

To examine whether persons who work with pigs are at high risks for HEV infection, 105 subjects (63 males, aged $32.0 \pm 15.2$ years; 42 females, aged 37.8 \pm 10.6 years), who had been handling pigs for more than three months in three pig farms in Accra, were recruited to participate in the study between January and May, 2008. The subjects' samples were assayed for the presence of anti-HEV IgG and IgM. There was no significant difference in age distribution, $p>0.05$. All the subjects were occupationally exposed to the pigs (feeding the pigs, cleaning barns, assisting the sows at birth and butchering on the farm). Of the subjects occupationally exposed to the pigs, $94.3 \%$ (99 out of 105) had been working in the same farm setting for less than six months whiles 5.7\% (6 out of $105)$ had been working in the same farm setting for more than six months.

None of the subjects tested positive for antiHEV IgG. Overall, the prevalence of anti- HEV IgM was $38.1 \%$ (40 out of 105). The sero-prevalence of antibodies to HEV detected in female pig handlers $(40.5 \%, 17$ out of 42$)$ was higher than in the male pig handlers $(36.5 \%, 23$ out of 63$)$. There was no significant difference between the two groups of pig handlers, $\mathrm{P}=0.83$. In bivariate analysis, no association was found between prevalence of infection and sex (odds ratio [OR] 0.85; 95\% confidence interval [CI], 0.379-1.885).

Anti-HEV prevalence was significantly higher $(\mathrm{P}=0.02)$ among persons who work with pigs and had been working in the same farm setting for less than six months compared to persons who work with pigs and had been working in the same farm setting for more than six months $(83.3 \%, 5$ out of 6 vs, $35.4 \%$, 
35 out 99 respectively). Persons who work with pigs and had been working in the same farm setting for less than six months had a nine fold higher risk of HEV infection as compared with those who had been working in the same farm setting for more than six months (OR 9.14; 95\% CI 1.02-81.38).

Among the persons who work with pigs, there was no significant difference $(\mathrm{p}=0.41)$ in anti-HEV reactivity between persons aged $>40$ years $(41.2 \%$, 26 out of 62 ) as compared to persons aged $<40$ years $(32.6 \%, 14$ out of 43$)$. However, in bivariate analysis, anti- HEV reactivity was positively associated with age (OR 1.49; CI 0.66-3.37).

Asimilar pattern of positive association (OR1.81; CI 0.67-4.81) of anti-HEV reactivity with education was found among persons who work with pigs. AntiHEV reactivity among persons who work with pigs with low level of education (primary or basic; $41.3 \%$, 33 out of 80) washigher than that of their counterparts with high level of education (secondary; 28\%, 7 out of 25). There was no significant difference between the two groups of pig handlers, $\mathrm{p}=0.25$.
Potential risk factors for infection were examined, to determine whether there were associations with anti-HEV prevalence. As shown in Table 1, persons who work with pigs and had water piped into their homes and / or on the farms, clean barns, assist sows at birth. Butcher pigs at the farm, and eat "khebab" (undercooked pork) had higher prevalence of HEV infection. Among the persons who work with pigs, the greatest risk of HEV seropositivity was strongly associated with those who had water piped into their homes and / or on the farms (OR 3.97; CI 0.44-90.83), and, to a lesser extent, those who had close contact with pigs, such as cleaning barns (OR 2.67; CI 0.48-19.30), assisting sows at birth (OR 2.10; CI0.63-7.34), and butchering pigs at the farm (OR 2.84; CI 1.057.92) (Table 1). Interestingly, there was no association (OR $0.73 ; \mathrm{Cl}$ 0.31 -1.70) of anti- HEV reactivity with persons who work with pigs who had reported having a history of a cut with blood-to-blood contact (Table 1).

Table 1

Odds ratio for HEV sero-positivity and corresponding 95\% confidence intervals according to risk factors among 105 occupational exposed pig handlers in Accra, Ghana

\begin{tabular}{|c|c|c|c|c|}
\hline \multirow[b]{2}{*}{ Characteristic } & \multirow[b]{2}{*}{ No. tested } & \multicolumn{3}{|c|}{ HEV sero-positivity } \\
\hline & & No. & $(\%)$ & OR $(95 \% \quad C I)$ \\
\hline \multicolumn{5}{|c|}{ Piped water in household } \\
\hline Yes & 98 & 39 & 39.8 & \\
\hline No & 7 & 1 & 14.3 & $3.97(0.44-90.83)$ \\
\hline \multicolumn{5}{|l|}{ Cleaning barns } \\
\hline Yes & 95 & 38 & 40.0 & \\
\hline No & 10 & 2 & 20.0 & $2.67(0.48-19.30)$ \\
\hline \multicolumn{5}{|c|}{ Assisting sows at birth } \\
\hline Yes & 85 & 35 & 41.2 & \\
\hline No & 20 & 5 & 25.0 & $2.10(0.63-7.34)$ \\
\hline \multicolumn{5}{|c|}{ Butchering at farm } \\
\hline Yes & 70 & 32 & 45.7 & \\
\hline No & 35 & 8 & 22.9 & $2.84(1.05-7.92)$ \\
\hline \multicolumn{5}{|c|}{ Eating undercooked pork( Khebab) } \\
\hline Yes & 97 & 38 & 39.2 & \\
\hline No & 8 & 2 & 25.00 & $1.93(0.33-14.68)$ \\
\hline \multicolumn{5}{|c|}{ Reported cut with blood-blood contact } \\
\hline Yes & 12 & & 33.3 & \\
\hline No & 28 & & 40.5 & $0.73(0.31-1.70)$ \\
\hline
\end{tabular}

In a multivariate analysis, HEV infection was independently associated with length of time a person has been working on the farm and having piped water into their homes and/or the farms. 


\section{DISCUSSION}

Ghana, an area of endemicity for viral hepatitis B and $C$, has never had an epidemic of hepatitis E. However, recent reports from the Gastroenterology Unit of the Department of Medicine and Therapeutics, Korle-Bu Teaching Hospital, Accra, Ghana indicate cases of acute hepatitis without a defined aetiology. Although the physicians did not estimate HEV or hepatitis A virus (HAV) antibodies in the patients' serum, based on clinical examinations, they speculated that HEV or HAV may be the causative pathogen. Since none of the patients had a disease-related history of travel to areas where HEV is endemic, it is quite likely that the transmission of HEV occurred in Ghana. Recent reports indicate that pigs are the main reservoirs for HEV transmission to humans (4-9) and that persons who work with pigs, such as veterinarians and pig handlers, are at increased risk of zoonotic infection (10-12). The purpose of this study was to determine the seroprevalence of HEV and also the correlates of HEV infection among persons who work with pigs in some selected farms in Accra, Ghana.

To our knowledge, this is believed to be the first study to determine the risk factors associated with HEV infection in persons who work with pigs in Ghana, and demonstrates the high prevalence of and the considerable potential for the transmission of HEV infection in pig farms in Ghana. Although there is no report from the Ministry of Health, indicating that Ghana is an endemic area for hepatitis E, this study found very high overall prevalence rates $(38.1 \%)$ of HEV antibody among persons occupationally exposed to pigs, suggesting the possibility of subclinical infections in the country. Similar seroprevalence rates to HEV were noted in human populations living in countries where HEV is not endemic (10-13). Further studies need to be done to isolate both the pig and human HEV strains in Ghana and determine the genomic relationshipbetween them if both the human and pig HEV are isolated.

The risk of HEV infection correlated with age, length of time employed, close contact with animals or animal waste, such as cleaning barns or assisting sows at birth, butchering pigs, and having piped water in homes and / or on the farms. In addition, levels of anti-HEV infection were higher among persons who work with pigs with low level of education.

The finding of higher HEV antibody prevalence in persons who work with pigs in Ghana is consistent withliterature, and is widely attributabletowork-related behaviours practised on the farm settings, although transmission of HEV has also been documented among individuals outside the farm setting and persons who arenotoccupationally exposed to pigs $(10-12,14,15)$. The overall seroprevalence of HEV infection among persons who work with pigs in Ghana (38.1\%) is higher (26.7\%) than the results of similar studies in persons who work with pigs in Taiwan (11) but comparable to the reported seroprevalence of $51.1 \%$ in persons who work with pigs inMoldova(10). Theincreased seroprevalence of HEV in persons who work with pigs (38.1\%) in Ghana suggests that HEV may be widespread in pig populations in the country and therefore reasonable to speculate that $\mathrm{HEV}$ may circulate in the general population. In addition, because the virus is transmitted through the faecal-oral route, transmission of HEV is greatly dependent on the sanitary conditions under which the pig handlers work. In Ghana, there are great social differences and sanitary conditions are quite precarious in many areas. The sanitary conditions at the work place during the period of study were very deplorable and all the farms were situated in densely populated areas where the animals share their habitat with humans. Of interest, persons who work with pigs that had water piped into their homes and / or on the farms had higher anti-HEV reactivity than those who do not have piped water into their homes and/or on the farms (39.8\% vs. $14.3 \%$, respectively; Table1). This finding is notsurprising since most of the water delivery pipes are broken and/or exposed and as such the water may have been easily contaminated with faecal effluent. Moreover, handwashing facilities are not easily available in the farms.

Another finding of interest reported herein in our study is that anti-HEV prevalence was significantly higher $(\mathrm{P}=0.02)$ among persons who had been working with pigsin thesamefarm setting for less than six months compared to those who had been working in the same farm setting for more than six months $(83.3 \%, 5$ out of 6 vs, 35.4\%, 35 out 99 respectively). The reason(s) for this disparity cannot be discerned from our study. However, it was observed that newly recruited individuals spend more time cleaning barns and assisting sows at birth, and this perhaps may have accounted for the high seroprevalence rate of HEV infection. Further studies need to be done to define the high prevalence of antiHEV antibodies in such population.

Strikingly, none of the pig handlers studied tested positive for antibodies to IgG anti- HEV. The presence of seropositive IgM anti-HEV usually indicates recent HEV infection (16) and may signify recent introduction of $\mathrm{HEV}$ into these farms. There is therefore the need to investigate other farms around these location to ascertain whether there had been any earlier infections among farm workers. There is also the need for further studies to define the clinical and epidemiological importance and pathogenesis of HEV infection in this population.

The small sample size and our inability to test for anti-HEV reactivity in pigs may be the limitations of this study. However, pigs may not be the only animal reservoir for HEV. That hepatitis $E$ has been reported in countries that do not traditionally raise pigs and that the high rate of seroprevalence of anti-HEV in the general population in areas where HEV is not endemic suggest the presence of other animal reservoirs for HEV. In fact, serum anti-HEV has been found in domestic animals such as rats, sheep, dogs, cats and 
may serve as reservoirs for the transmission of human hepatitis E (17-20). The detection and prevalence of $\mathrm{HEV}$ infection in persons who work with pigs in Ghana may reflect the prevalence of HEV infections among domestic animals in the country. A growing body of data supports the zoonotic nature of HEV infection (17 -20). Further studies need to be done to define clearly the natural history of HEV infection and transmission in Ghana in order to effectively control and prevent HEV zoonosis.

The results reported herein have significant implications for veterinarians, public health officials, persons who work with pigs and farm managers, and suggest urgent need for the introduction of policies to prevent the transmission of HEV on the farms and the general population. These policy strategies must include increasing education of persons who work with pigs about HEV testing and prevention of infected pig handlers. The implementation of a $\mathrm{HEV}$ infection prevention programme in pig farms in Ghana should be seen as an opportunity to improve the health status of the infected persons who work with pigs and to prevent further transmission of $\mathrm{HEV}$, within and without the farm settings.

In conclusion, our results suggest that persons who work with pigs who are occupationally exposed to pigs are at increased potential risk of zoonotic HEV infection. Thehigh prevalence of HEV infection found in persons who work with pigs in Ghana suggests the urgent need for the introduction of some of the range of effective preventive strategies employed in pig farm settings elsewhere.

\section{ACKNOWLEDGEMENTS}

We are grateful to the farm managers and the pig handlers. This study was supported with funds from the University of Ghana Research and Conferences Committee.

\section{REFERENCES}

1. Emerson, S. U., and Purcel, R. H. Hepatitis E virus. Rev. Med. Virol. 2003; 13: 145-154.

2. Irshad,M.HepatitisEvirus: anupdateonitsmolecular, clinical and epidemiological characteristics. Intervirol. 1999; 42: 252-262.

3. Aggarwal, R. and Krawczynski, K. Hepatitis E: An overview and recent advances in clinical laboratory research. J. Gastroenterol. Hepatol. 2000; 15: 9-20.

4. Meng, X. J., Purcell, R. H., Halbur, P. G., et al. A novel virus in swine is closely related to the human hepatitis E virus. Proc. Natl. Acad. SciUSA. 1997; 94: 9860-9865.

5. Erker,J.C., Desai, S.M.,Schaudler, G.G., et al. A hepatitis E virus variant from the United States: molecular characterisation and transmission in cynomolgus macaques. J. Gen. Virol. 1999 ; 80: 681-690.

6. Lu, L., Li, C. H. and Hagedorn, C. Phylogenetic analysis of global hepatitis E virus sequences: genetic diversity, subtypes and zoonosis. Rev. Med. Virol. 2006; 16: 5- 36.

7. Zhang, Y., Ge, B., Zhang, I., Guo, G., et al. Swine as principal reservoir of hepatitis virus that infects humans in Eastern China. J. Infect. Dis. 2006; 193: 1643-1649.

8. Choi, I. S., Kwon, H. I., Shin, N. R. and, Yoo, H. S. Identification of swine hepatitis $\mathrm{E}$ virus (HEV) and prevalence of anti- HEV antibodies in swine and human populations in Korea. J. Clin. Microbiol. 2003; 41: 3602-3608.

9. Takahashi, M., Ishikawa, T. and Okamoto, H. Identification of genotype III swine hepatitis E virus that was isolated from a Japanese pig born in 1990 and that is most closely related to Japanese isolates of human hepatitis E virus. J. Clin. Microbiol. 2003; 1: 1342-1343.

10. Drobeniuc,J.,Favorov,M.O.,Shapiro,C.N., etal.Hepatitis E virus antibody prevalence among persons who work with swine. J. Infect. Dis. 2000; 184: 1594-1597.

11. Hsieh, S.Y., Meng, X. J., Wu, Y. H., et al. Identity of a novel swine hepatitis $E$ virus in Taiwan forming a monophyletic group with Taiwan isolates of human hepatitis E virus. J. Clin. Microbiol. 1999; 37: 3828- 3834.

12. Meng, X. J., Wiseman, B., Elvinger, F. et al. Prevalence of antibodies to hepatitis $E$ virus in veterinarians working with swine and in normal blood donors in the United States of America and other countries. J. Clin. Mirobiol. 2002; 40: 117-122.

13. Lin, C. C., Wu, J. C., Chang, T. T., et al. Diagnostic value of immunoglobulin $\mathrm{G}$ (IgG) and IgM anti hepatitis E virus (HEV) tests based on HEV RNA in the area where hepatitis E is not endemic. J. Clin. Microbiol. 2000; 38: 3918-3925.

14. Fix, A. D., Abdel-Harnid, M., Purcell, R. H., et al. Prevalence of antibodies to hepatitis $\mathrm{E}$ in rural Egyptian Communities. Am. J. Trop. Med. Hyg. 2000; 62: 519-523.

15. Mast, E. E., Kuramoto, I. K., Favorov, M. O., et al. Prevalence of risk factors for antibodies to hepatitis E virus seroactivity among blood donors in Northern California. J. Infect. Dis. 1997; 176: 34-40.

16. Lin, C.C., Lok, A. S. F., Kwan, W. K., et al. Seroepidemiological survey of hepatitis $\mathrm{E}$ in Hong Kong by recombinant-based enzyme immunoassay. Lancet. 1992; 340: 1205-1208.

17. Clayson, E.T., Snitbhan, R., Ngarmpochana, M., et al. Evidence of the hepatitis E virus (HEV) is a zoonotic: detection of natural infection among swine, rats, chicken in an area endemic for human disease, 1996; p.329-355.In:Busson, Y., Coursaget, P., Kane, M. (ed.), Enterically transmitted hepatitis viruses. La Simane, Joue-Ies-Tours, France.

18. Favorov, M. O. and Margolis, H. S. Hepatitis E virus infection: an entrically transmitted cause of hepatitis, 1-6. In: Scheid, W. M., Craig, W. A. and Hughes, J. M., (eds.), Emerging infections 3. Washington DC. 1999; Amer. Soc. Microbial. Press.

19. Purcell, R.H. and Emerson, S. U. Animal models of hepatitis A and E. ILAR. J. 2001; 42: 161-177.

20. Thomas, D. I., Yarbough, P. O. and Vlahov, D. Seroreactivityof hepatitis $\mathrm{E}$ virus in areas where the disease is not endemic. J. Clin. Microbiol. 1997; 35: 1244-1247. 